\title{
Enhanced pseudo Zernike moments in face recognition
}

\author{
Ying-Han Pang, ${ }^{\text {a) }}$ Andrew Teoh B. J., and David Ngo C. L. \\ Faculty of Information Science and Technology, Multimedia University \\ Jalan Ayer Keroh Lama, 75450 Melaka, Malaysia \\ a)yhpang@mmu.edu.my
}

\begin{abstract}
This paper presents an approach to boost the performance of pseudo Zernike moments in face recognition. This approach is a hybrid of a kernel trick, discriminant function and pseudo Zernike moments (PZM), namely as Kernel-based Fisher Pseudo Zernike Moments (KFPZM). KFPZM maps the moment-based features into a high dimensional feature space via kernel function for disclosing the underlying variables which carry significant information about the image. Then, it performs discriminant analysis onto the mapped features to enhance the discrimination power via Fisher's Linear Discriminant (FLD). Experimental results show that the proposed method outperforms the sole PZM and the integrated FLD with PZM methods, achieving recognition rate of $98.11 \%$ and $93.03 \%$ in the face databases with facial expression variations and illumination variations, respectively.
\end{abstract}

Keywords: Face recognition, kernel, Fisher's Linear Discriminant, pseudo Zernike moments

Classification: Science and engineering for electronics

\section{References}

[1] J. Haddadnia, K. Faez, and P. Moallem, "Neural Network based Face Recognition with Moment Invariants," Proc. Int. Conf. Image Processing, vol. 1, pp. 1018-1021, Oct. 2001.

[2] Ying-Han Pang, A. Teoh, and D. Ngo, "Face Authentication System using Pseudo Zernike Moments on Wavelet Subband," IEICE Electron. Express, vol. 1, no. 10, pp. 275-280, Aug. 25, 2004.

[3] M. H. Yang, "Kernel Eigenfaces vs. Kernel Fisherfaces: Face Recognition Using Kernel Methods," Proc. 5th IEEE Int. Conf. Automatic Face and Gesture Recognition, pp. 205-211, May 2002.

[4] Q. S. Liu, R. Huang, H. Q. Lu, and S. D. Ma, "Face Recognition Using Kernel Based Fisher Discriminant Analysis," Proc. 5th IEEE Int. Conf. Automatic Face and Gesture Recognition, pp. 187-191, May 2002.

[5] R. Mukundan and K. R. Ramakrishnan, Moment Functions in Image Analysis - Theory and Applications, World Scientific Publishing, Singapore, 1998.

[6] C. H. Teh and R. T. Chin, "On Image Analysis by the Methods of Moments," IEEE Trans. Pattern Anal. Machine Intell., vol. 10, pp. 496-512, July 1988.

[7] A. Khotanzad, "Invariant Image Recognition by Zernike Moments," IEEE Trans. Pattern Anal. Machine Intell., vol. 12, pp. 489-497, May 1990. 


\section{Introduction}

Moment functions model an image into a compact subspace represented by moment bases. Small number of moments is sufficient to describe most of the image structure. Therefore, Haddadnia et al. employed moment functions in face recognition [1]. [1] and [2] reported that pseudo Zernike moments show the recognition rate of $98.73 \%$ by using radial basis function neural network classifier, and $93.46 \%$ using Euclidean distance, respectively. Indeed, the performance of moment functions can be further improved by integrating a kernel and discriminant functions into moment analysis without resorting any complex classifier, likes neural network. Thus, in this paper, Kernelbased Fisher Pseudo Zernike Moments (KFPZM) method is proposed for face recognition.

Inspired by the ideas of Kernel PCA [3] and Kernel LDA [4], KFPZM combines a kernel trick with pseudo Zernike moments (PZM) that coupled with Fisher's Linear Discriminant (FLD). This integration not only can account for the variables that implicitly underlie on the image structure, but also enhances the discrimination power of the moments. Fig. 1 illustrates the overview of KFPZM. In this method, the image data is firstly transformed into a set of moment-based features via PZM computation. Then, these features are further mapped into a higher dimensional feature space via kernel function to disclose the underlying variables which carry more significant and expressive information about the image. Finally, the mapped features are input into FLD to further enhance the discrimination power of the features by maximizing the between-class scatter and minimizing the within-class scatter.

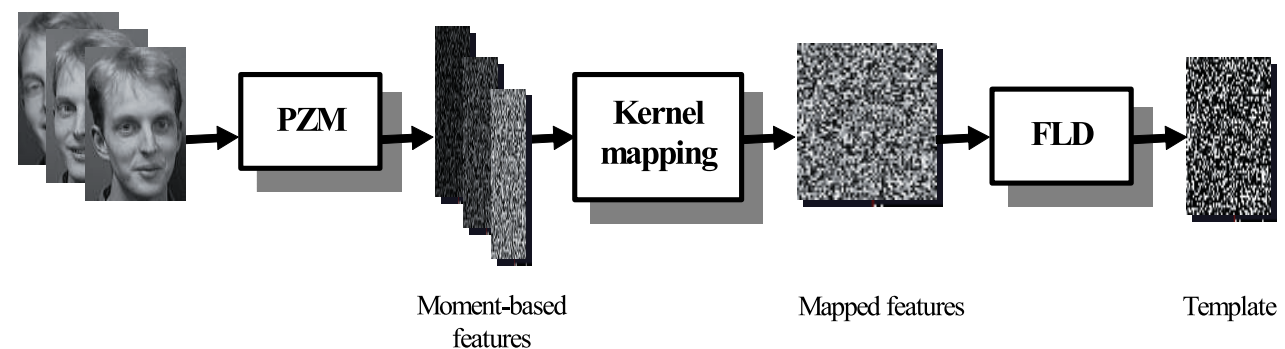

Fig. 1. Overview of KFPZM method.

\section{Pseudo Zernike moments}

The two-dimensional pseudo Zernike moments of order $p$ with repetition $q$ of image intensity function, $f(r, \theta)$, are defined as $[5,6]$ :

$$
P Z_{p q}=\frac{p+1}{\pi} \int_{0}^{2 \pi} \int_{0}^{1} V_{p q}(r, \theta) f(r, \theta) r d r d \theta
$$

where Zernike polynomials $V_{p q}(r, \theta)$ are defined as:

$$
V_{p q}(r, \theta)=R_{p q}(r) e^{-\hat{j} q \theta} ; \quad \hat{j}=\sqrt{-1}
$$


and $r=\sqrt{x^{2}+y^{2}}, \theta=\tan ^{-1}\left(\frac{y}{x}\right),-1<x, y<1$

The real-valued radial polynomials are defined as:

$$
R_{p q}(r)=\sum_{s=0}^{p-|q|}(-1)^{s} \frac{(2 p+1-s) !}{s !(p+|q|+1-s) !(p-|q|-s) !} r^{p-s}
$$

and $0 \leq|q| \leq p, p \geq 0$

In this study, the considered features are the magnitude of $P Z_{p q}$, abbreviated as $P Z_{p q, m a g}$, due to their rotation invariant property; the phase information is omitted as the influence of phase information is rather insignificant especially when high order moments are included [7].

\section{Kernel-based Fisher Pseudo Zernike Moments}

PZM transforms face images into moment-based feature vectors, $P Z_{p q, m a g}$, in input space, $R$. Then, this input data is projected into a higher dimensional implicit feature space, $F$, via nonlinear mapping, $\Phi: P Z_{p q, m a g} \in R \rightarrow f \in F$. Next, FLD is performed onto the mapped features in order to maximize the between-class scatter and minimize the within-class scatter. It is no need to compute $\Phi$ explicitly but just compute the inner product of the two vectors, $f$, in $F$ with a kernel function:

$$
k\left(P Z_{p q, m a g}, P Z_{p q, m a g k}\right)=\left(\Phi\left(P Z_{p q, m a g ~ j}\right) . \Phi\left(P Z_{p q, m a g k}\right)\right)
$$

In feature space, $F$, the between- and within-class scatter, $\hat{S}_{b}$ and $\hat{S}_{w}$ respectively, are defined as:

$$
\begin{gathered}
\hat{S}_{b}=\sum_{i=1}^{C} n_{i}\left(\mu_{i}-\mu\right)\left(\mu_{i}-\mu\right)^{T} \\
\hat{S}_{w}=\sum_{i=1}^{C} \sum_{k=1}^{n_{i}}\left(\Phi\left(P Z_{p q, \text { mag } i k}\right)-\mu_{i}\right)\left(\Phi\left(P Z_{\text {pq,mag } i k}\right)-\mu_{i}\right)^{T}
\end{gathered}
$$

where

$$
\begin{aligned}
& \text { class mean, } \quad \mu_{i}=\frac{1}{n_{i}} \sum_{k=1}^{n_{i}} \Phi\left(P Z_{p q, \text { mag } k}\right) \\
& \text { grand mean, } \quad \mu=\frac{1}{n} \sum_{j=1}^{n} \Phi\left(P Z_{p q, \text { mag } j}\right)
\end{aligned}
$$

Note that $\mathrm{C}$ is the number of classes, $n$ is the total number of mapped samples, $n_{i}$ is the number of mapped samples in $i$ th class and $P Z_{p q, m a g} i k$ is the $k$ th moment-based feature vector in $i$ th class.

To perform FLD in $F$, it is equal to maximize expression [4],

$$
J(w)=\frac{w^{T} \hat{S}_{b} w}{w^{T} \hat{S}_{w} w}
$$

Because any solution $w \in F$ must lie in the span of all the samples in $F$, there exist coefficients $\alpha_{j}, j=1,2, \cdots, n$, such that

$$
w=\sum_{j=1}^{n} \alpha_{j} \Phi\left(P Z_{p q, m a g j}\right)
$$


By expression (10), the projection of each class mean $\mu_{i}$ onto $w$ can be written

$$
\begin{aligned}
& w^{T} \mu_{i}=\alpha^{T} \sum_{j=1}^{n} \Phi\left(P Z_{p q, \text { mag } j}\right) \frac{1}{n_{i}} \sum_{k=1}^{n_{i}} \Phi\left(P Z_{p q, \text { mag } k}\right) \\
&= {\left[\begin{array}{c}
\frac{1}{n_{i}} \sum_{k=1}^{n_{i}} \Phi\left(P Z_{p q, \text { mag } 1}\right) . \Phi\left(P Z_{p q, \text { mag } k}\right) \\
\frac{1}{n_{i}} \sum_{k=1}^{n_{i}} \Phi\left(P Z_{p q, \text { mag } 2}\right) . \Phi\left(P Z_{p q, \text { mag } k}\right) \\
\vdots \\
\frac{1}{n_{i}} \sum_{k=1}^{n_{i}} \Phi\left(P Z_{p q, \text { mag } n}\right) . \Phi\left(P Z_{p q, \text { mag } k}\right)
\end{array}\right] } \\
&= {\left[\begin{array}{c}
\frac{1}{n_{i}} \sum_{k=1}^{n_{i}} k\left(P Z_{p q, \text { mag } 1}, P Z_{p q, \text { mag } k}\right) \\
\frac{1}{n_{i}} \sum_{k=1}^{n_{i}} k\left(P Z_{p q, \text { mag } 2}, P Z_{p q, \text { mag } k}\right) \\
\vdots \\
\frac{1}{n_{i}} \sum_{k=1}^{n_{i}} k\left(P Z_{p q, \text { mag } n}, P Z_{p q, \text { mag } k}\right)
\end{array}\right] } \\
&=\alpha^{T} M_{i}
\end{aligned}
$$

It follows that $w^{T} \hat{S}_{b} w=\alpha^{T} K_{b} \alpha$, where $K_{b}=\sum_{i=1}^{C} n_{i}\left(M_{i}-M\right)\left(M_{i}-M\right)^{T}$, and $M=\left[\begin{array}{c}\frac{1}{n} \sum_{k=1}^{n} k\left(P Z_{p q, \text { mag } 1}, P Z_{p q, \text { mag } k}\right) \\ \vdots \\ \frac{1}{n} \sum_{k=1}^{n} k\left(P Z_{p q, \text { mag } n}, P Z_{p q, \text { mag } k}\right)\end{array}\right]$; similar to within-class scatter, $w^{T} \hat{S}_{w} w=\alpha^{T} K_{w} \alpha$, where $K_{w}=\sum_{i=1}^{C} \sum_{k=1}^{n_{i}}\left(\xi_{k}-M_{i}\right)\left(\xi_{k}-M_{i}\right)^{T}$, and $\xi_{k}=$ $\left(k\left(P Z_{p q, \operatorname{mag} 1}, P Z_{p q, \text { mag } k}\right), k\left(P Z_{p q, \operatorname{mag} 2}, P Z_{p q, \text { mag } k}\right), \cdots, k\left(P Z_{p q, m a g n}\right.\right.$, $\left.\left.P Z_{p q, \text { mag } k}\right)\right)^{T}$ The kernel trick used in this study is Gaussian function:

$$
k(x, y)=\exp \left(-\|x-y\|^{2} / \sigma\right)
$$

where $\sigma$ is the Gaussian parameter.

\section{Experiment}

We evaluate the performance of KFPZM method by using two face databases: Essex Face94 with facial expression variations and Essex Face95 with illumination variations. These databases are made publicly available at URL http://cswww.essex.ac.uk/mv/allfaces/index.html. In both databases, 10 face samples are used for training and another 10 for testing for each subject. There are 100 and 72 face classes in Face94 and Face95 databases. Comparative analysis is carried out among PZM, integrated FLD and PZM (MF) and KFPZM methods by using Euclidean distance as classifier. 
Excessive higher order moments comprise redundant and unwanted information, such as noise, and these high frequency variables may influence the discrimination power. Thus, the performance of KFPZM method with different moment orders is evaluated to justify the statement.

\section{Results and discussions}

Fig. 2 shows the recognition rates of the PZM, MF and KFPZM methods in both face databases. From Fig. 2, we can see that KFPZM with kernel parameter, $\sigma=200$ (represented by KMF : G_par = 200) obtain the highest recognition rate of $98.11 \%$ (with moment length 160) in Face94 database and 93.03\% (with moment length 140) in Face95 database. This demonstrate that the integration of kernel and FLD with moments helps enhance the discrimination power by disclosing the underlying significant image features and maximizing the between-class scatter while minimizing the within-class scatter.

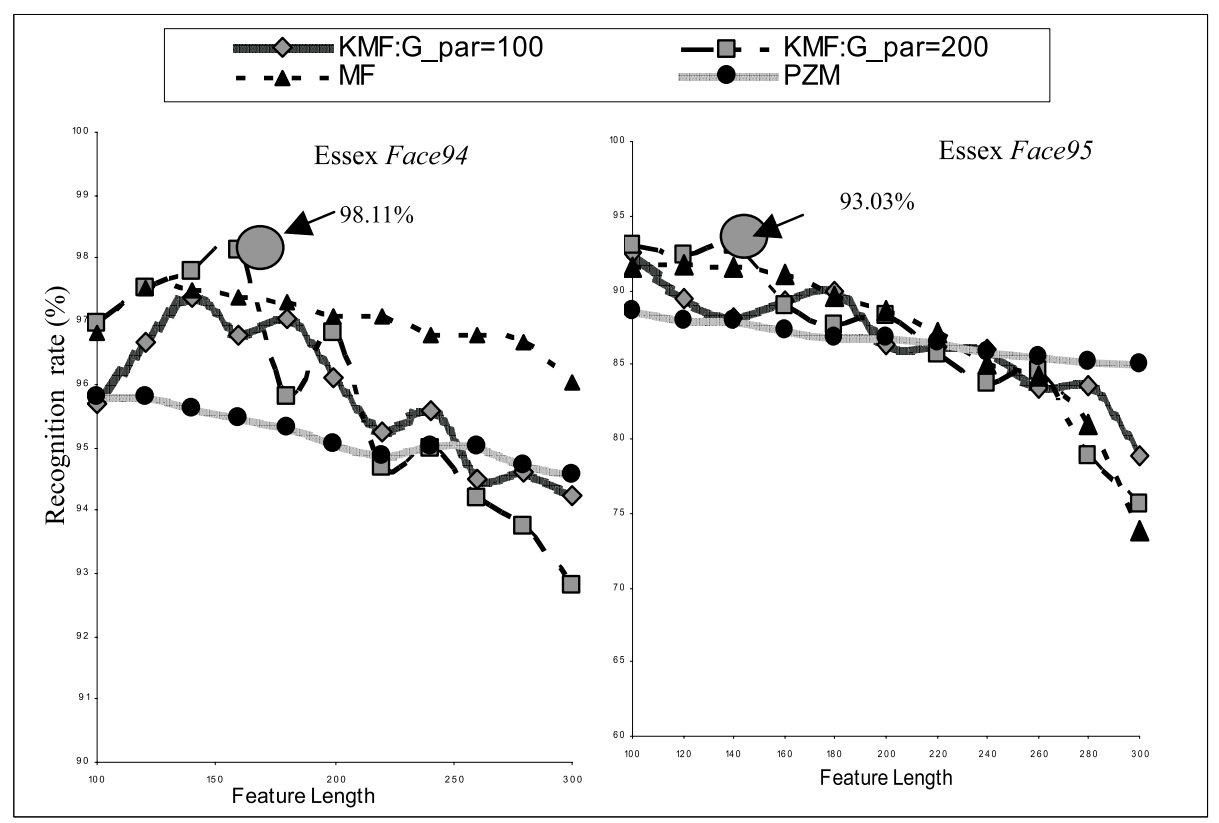

Fig. 2. Recognition rates of PZM, MF and KFPZM methods.

However, KFPZM obtains poorer result when higher order moments are included. This is because higher order moments bring in high frequency information and redundant data that affect the separability of KFPZM. The statement is further justified in Table I that KFPZM method with lower order moments performs better than that with higher order moments.

\section{Conclusion}

A new approach by using pseudo Zernike moments integrated with kernel and discriminant functions has been presented in this paper in order to boost the recognition performance of pseudo Zernike moments on face images. This 
Table I. Performance on different order moments in KFPZM method.

\begin{tabular}{|c|c|c|c|c|c|}
\hline $\begin{array}{c}\text { Face } \\
\text { database }\end{array}$ & $\begin{array}{c}\text { Kernel } \\
\text { parameter, } \sigma\end{array}$ & $\begin{array}{l}\text { Moments } \\
\text { in order }\end{array}$ & FAR $(\%)$ & $\operatorname{FRR}(\%)$ & TSR (\%) \\
\hline \multirow{10}{*}{ Face94 } & \multirow{5}{*}{100} & 1 to 100 & 4.30 & 4.30 & 95.70 \\
\hline & & 100 to 200 & 10.43 & 10.40 & 89.57 \\
\hline & & 200 to 300 & 17.93 & 18.10 & 82.06 \\
\hline & & 300 to 400 & 21.98 & 21.70 & 78.03 \\
\hline & & 400 to 500 & 24.61 & 24.40 & 75.40 \\
\hline & \multirow{5}{*}{200} & 1 to 100 & 3.02 & 3.00 & 96.98 \\
\hline & & 100 to 200 & 12.08 & 12.10 & 87.92 \\
\hline & & 200 to 300 & 16.33 & 16.4 & 83.67 \\
\hline & & 300 to 400 & 23.55 & 23.40 & 76.46 \\
\hline & & 400 to 500 & 24.30 & 24.20 & 75.70 \\
\hline \multirow{10}{*}{ Face95 } & \multirow{5}{*}{100} & 1 to 100 & 7.50 & 7.50 & 92.50 \\
\hline & & 100 to 200 & 17.14 & 17.50 & 82.84 \\
\hline & & 200 to 300 & 26.16 & 26.25 & 73.84 \\
\hline & & 300 to 400 & 27.72 & 27.78 & 72.28 \\
\hline & & 400 to 500 & 29.61 & 29.44 & 70.40 \\
\hline & \multirow{5}{*}{200} & 1 to 100 & 6.94 & 6.95 & 93.04 \\
\hline & & 100 to 200 & 14.88 & 14.86 & 85.12 \\
\hline & & 200 to 300 & 20.63 & 20.70 & 79.37 \\
\hline & & 300 to 400 & 31.06 & 31.25 & 68.93 \\
\hline & & 400 to 500 & 32.51 & 32.36 & 67.50 \\
\hline
\end{tabular}

method is called Kernel-based Fisher Pseudo Zernike Moments which hybrids the Gaussian function and Fisher's Linear Discriminant with pseudo Zernike moments. In this approach, the kernel function maps the momentbased features into a high dimensional space so that the underlying informative features can be revealed. Fisher's Linear Discriminant is then performed onto the mapped features to maximize the between-class scatter and minimize the within-class scatter for increasing the separability of the features. Experimental results show that the proposed method achieves superior performance than the PZM and MF methods.

\section{Acknowledgments}

The authors thank Vision Group of Essex University for face database provisions. 